\title{
The effects of age interval of the stimuli and sexual orientation of the participants on face recognition among Filipino college students
}

Dullas, Angelo R.

University of Santo Tomas, Central Luzon State University, Philippines (dullas.angelo@yahoo.com) Tolentino, Marvin N. $\bowtie$

Central Luzon State University, Philippines (tolentinomarvin@gmail.com)

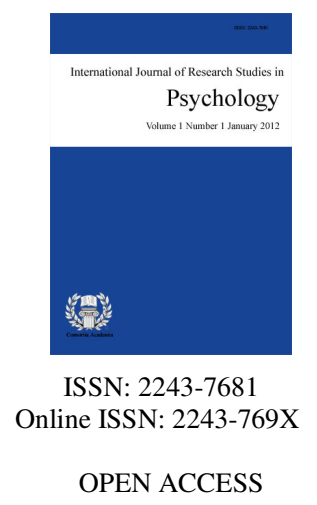

Received: 28 February 2016

Revised: 9 April 2016 DOI: $10.5861 /$ ijrsp.2016.1475

\section{Abstract}

For the past years, studies on face recognition were focused on the effects and influence of illumination, pose, and angle to the performance, which, in fairness, has been proven by different research works as the dominant factor of adding complexity to face recognition across age progression observed through photographs, specifically headshots (Ling, Soatto, Ramanathan, \& Jacobs, 2007; Ramanathan \& Chellappa, 2006; Ramanathan \& Chellappa, 2005). However, studies on face verification and recognition with time lapses between pairs of photographs and the interaction on the sexual orientation of the identifier was somehow defocused on the factor of effects of age progression or the time interval between pairs of facial images on face recognition. Thus, the researchers aimed to resolve the gap on the prevailing literatures on face recognition by finding the effects of age interval of the stimuli and sexual orientation of the participants on face recognition using Filipino college students as sample. Results showed that the greater the age gaps between the pair of images, the lesser the chance of identifying it. In addition, it is also not important to consider whether identifier is male or female in terms of recognizing picture because based from the result of the experiment, sexual orientation does not have an effect on the performance of the participants in face recognition. The study was guided by the theory of Biederman (1987) and Recognition By Components (RBC) to analyze the findings.

Keywords: age gap; face recognition/identification; Filipino college students; sexual orientation 


\section{The effects of age interval of the stimuli and sexual orientation of the participants on face recognition among Filipino college students}

\section{Introduction}

Face recognition across ages is an essential predicament and has many implications. Passport verification, image retrieval, surveillance, identity check, and renewal of IDs are among the many (Ling, Soatto, Ramanathan, \& Jacobs, 2007). It is also a way of helping criminal cases thru proper identification of the eyewitness on the culprit or suspect/s. For the previous years, however, studies on face recognition through photographs were focused on the effects and influence of illumination, pose, and angle to the performance, which, in fairness, has been proven by different research works as the dominant factor of adding complexity to face recognition across age progression observed through photographs, specifically headshots (Ling et al., 2007; Ramanathan \& Chellappa, 2006; Ramanathan \& Chellappa, 2005). Conversely, since there are already an overwhelming number of research works about the former, studies on face verification and recognition with time lapses between pairs of photographs and the interaction on the sexual orientation of the identifier was somehow defocused on the factor of how age progression or the time interval between pairs of facial images.

Although different studies on how recognition performance varies with increasing time lapses between images have used a variety of techniques on identifying the influence of age progression to face/ or image identification. Such studies have turned out that aside from the already-proven nuisance variable of illumination, expression, and angle, biological factors that naturally occur as one develops physically through ages are also significant in adding difficulty to face recognition. Face recognition and detection has been widely studied for several decades. A lot of work has been done to handle the problem under different conditions, including lighting, pose, expression, and others while in comparison, face verification across ages is far less studied. In this section, however, related studies about face recognition are reviewed.

\section{Review of Related Literature}

\subsection{Factors in face recognition across age progression}

As age progression is inevitable, capturing one's present image is able through photography. Hence, further changes as human age is best traced through a series of photographs and a sequence of these facial developments can be recognized. Because of the different changes that occur on one's life span, face recognition is often made intricate and complicated by different factors. The most rational factor is the cycle of biological developments that humans undergo. In recent studies about face verification across age progression, the changes in one's skull structure are outstandingly distinguished. A study by Ramanathan and Chelleppa (2005) confirms that aging effects on human faces manifest in different forms in different ages. Their study backs up that while aging effects in younger years are manifested more in terms of changes in the cranium (upper section of the skulls)'s shape, they are manifested in older years in terms of skin artifacts. The cranium, being also defined as the skull excluding the mandible (jawbone), and which helps define one's head and facial stature, grows over a specific time through a phase and occurrence commonly called as the craniofacial growth or the skeletal maturation.

Ling, Soatto, Ramanathan, and Jacobs (2007) also affirms of what craniofacial growth studies validate that face profiles such as boundary shapes, eye locations, and the like, are relatively stable for people older than 18 years and that such development remains even until the age of 65. To support, researchers from psychophysics studied craniofacial growth as a result of internal forces acting on the human cranium (Chellappa, 2007). In line with this, there are sequences of changes in the shape of the cranium from infancy to teenage or pubertal growth periods, which is greatly of vertical facial development. Moreover, facial growth is characterized during 
The effects of age interval of the stimuli and sexual orientation of the participants on face recognition

formative years ( $0-18$ years). Hence, from zero to eighteen years face recognition across age progression has an added difficulty as the facial features naturally experience drastic changes.

Whilst changes occur greatly because of craniofacial growth in younger years, there also are facial variations during one's adult years. They are manifested more in terms of wrinkles and other major or minor changes in the skin texture. Also, facial features drift in a coherent pattern due to factors like sagging of muscles and weight gain or loss (Chellappa, 2007, p. 44). Apart from biological factors, factors such as climatic conditions, ethnicity, mental stress, etc., were also factors that often attributes in playing a role in the process of aging (Ramanathan \& Chelleppa, 2006, p. 3349).

Before facial aging effects became another interest of study, it had been proven that face recognition systems are sensitive to factors such as illumination and pose. It has then been a common fact that such nuisance factors are a dominant feature that brings or adds difficulty to age progression. Most of the previous studies use passport pictures that vary across ages with the same sets of people (those who need to renew their passport after a certain period of time, which usually takes years in interval) as database. Researchers have figured out that through the long time interval, changes in photography lighting poses or angle of subjects greatly influences facial recognition and verification. Hence, these factors are nuisance variables that should either be avoided or made equal among subjects so the difficulty in face recognition of a previous and future person's image will be minimized.

\subsection{Age gap or interval as a factor in face recognition}

In a study conducted by Ling et al. (2007), the recognition performance variation along the increasing time (year) lapses between images of persons were considered. Through the empirical research of passport photos that had different intervals (old and recent photo sets of passport holders), it was found out that the difficulty in recognition is saturated after the gap of the old photo and of the recent one is larger than four up to ten years. Supporting this study is that of Ramanathan and Chelleppa (2005) which had a result that misclassification of facial images across age progression is with the average age difference or interval of 7.4 years, which is almost the focal point of the given extra difficulty interval by Ling et al. (2007)'s study. Moreover, literature tells that as the age difference between pairs of images [with age intervals] increases, the proportion of images with high similarity scores (if there are participants assigned to match the facial images) decreases.

\subsection{Characteristics of participants as a factor in face recognition}

Studies on how recognition performance varies with given time lapses between images report that some characteristics of the participants or the ones who will be recognizing the facial images or photographs (or even real human faces) are significantly related to their identification performance. Such factors are their age, gender, and ethnicity or race. A study by Sporer (as cited in Anastasi \& Rhodes, 2005) discusses that individuals may acquire expertise for identifying faces from their own age group based from his in-group/out-group model of face recognition. Sporer's in-group/out-group model of face recognition (IOM) is affirmed by a previous study by Mason (1986) where he proved his hypothesis that people would remember faces most like their own as scientifically valid and correct as young subjects tends to make few errors with young faces and old subjects tend to make fewer errors with old faces. The IOM also covers issues on gender sensitivity of face recognition activities, even across age progression. Hence, as females are more likely to recognize significantly facial progression of female images, males also do with male facial images.

Moreover, in terms of component versus general approaches on face recognition, Heisele et al. (2003) suggests that component system of face recognition outperformed general based system of face recognition. These findings, in relation to the current study led the researcher to used Biederman (1987) Recognition By Components (RBC) Theory. The assumption of RBC relies on the idea that the perceptual recognition of the object or image is sectioned and arranged into simple geometric components (such as blocks, cylinders, wedges, 
and cones) called geons (Biederman, 1987). Thus, recognition relies not on the complete object but the object's components which are combinations of the geons.

\subsection{Current Study}

Based on the prevailing literature, studies on face recognition through photographs were focused on the effects and influence of illumination, pose, and angle to the performance, which, in fairness, has been proven by different research works as the dominant factor of adding complexity to face recognition across age progression observed through photographs, specifically headshots (Ling, et al., 2007; Ramanathan, \& Chellappa, 2006; Ramanathan, \& Chellappa, 2005). However, studies on face verification and recognition with time lapses between pairs of photographs and the interaction on the sexual orientation of the identifier was somehow defocused on the factor of how age progression or the time interval between pairs of facial images. Thus, the researcher aimed to resolving the gap between the prevailing literatures on face recognition through the current study.

This study on face recognition is significant as developing face recognition systems that are robust to age progression would enable the successful deployment of face recognition models. Such systems would be highly beneficial to security institutions, forensic investigations, ID processing, and the like. Further, verifying face images across age progression would make the necessity of periodically updating large face databases with more recent images a lot easier and convenient.

This study sought to answer the following questions: a) What are the mean scores and standard deviation values of the participants in picture recognition according to age gap conditions? 1 year old picture paired to 21 years old picture; 7 years old picture paired to 21 years old picture; 12 years old picture paired to 21 years old picture; b) What are the mean scores and standard deviation values in picture recognition according of the participants according to sexual orientation? c) Are there significant differences on the three treatment conditions of the study? d) Is there significant difference in terms of sexual orientation of the participants in picture recognition/identification? e) Is there an interaction effect between the treatment level - that is the picture gap or interval years in identifying picture and the sexual orientation of the participants?

On the aspect of research hypothesis, it is assumed that a) there are significant differences on the three treatment conditions; b) there is significant difference on sexual orientation of the participants in terms of recognizing picture; and c) there are interaction effects between the treatment levels - that is the picture gap or interval in years in identifying pictures and the sexual orientation of the participants.

\section{Method}

\subsection{Design}

The design of the study will be a 3 x 2 between subjects Analysis of Variance. This is further substantiated by the figure below. The picture gap and sex determine the levels. Three levels on picture gap such as $1 \mathrm{y} / \mathrm{o}$ picture paired to $21 \mathrm{y} / \mathrm{o}, 7 \mathrm{y} / \mathrm{o}$ paired to $21 \mathrm{y} / \mathrm{o}$, and $12 \mathrm{y} / \mathrm{o}$ paired to $21 \mathrm{y} / \mathrm{o}$ is presented while male and female determines the sex of the participants.

\subsection{Variables and Operational Definitions}

Independent Variable - Studies on how face recognition performance varies with given time lapses between images report that some characteristics of the participants or the ones who will be recognizing the facial images or photographs (or even real human faces) are significantly related to their identification performance. And one of these factors is the age (Anastasi \& Rhodes, 2005). In this study, the independent variable is the age interval of the stimulus which defined by the researcher as the picture gap. Picture gap - this is the interval in years of the 
The effects of age interval of the stimuli and sexual orientation of the participants on face recognition

stimulus. It has three (3) treatment levels: a.1 - $1 \mathrm{y} / \mathrm{o}$ picture of a female to be paired to four pictures of $21 \mathrm{y} / \mathrm{o}$ female pictures a.2 - $7 \mathrm{y} / \mathrm{o}$ picture of a female to be paired to four pictures of $21 \mathrm{y} / \mathrm{o}$ female pictures, a. $3-12 \mathrm{y} / \mathrm{o}$ picture of a female to be paired to four pictures of 21 .

Dependent Variable - Picture identification/recognition is the dependent variable of the study. It is defined as what the researchers' measure base on the scores tabulated from the performance of the participants in identifying the right picture. It is also defined as the target age as used in earlier studies in face recognition (Lamont, Stewart-Williams, \& Podd, 2005). Studies on how face recognition performance varies with given time lapses between images report that some characteristics of the participants or the ones who will be recognizing the facial images or photographs (or even real human faces) are significantly related to their identification performance. And of these factors is the age (Anastasi \& Rhodes, 2005).

\subsection{Participants}

Samples or participants of this study are the higher year students of the College of Arts and Sciences (CAS), Central Luzon State University. Participation in this study was agreed upon by the researcher and the participants. The participants were given a little description of the study and were asked for their compliance. The male and female participants who agreed were invited to the venue of the experiment. A total of 56 students (three males, three females per course) from the nine courses (namely AB Psychology, AB Social Sciences, AB Development Communication, AB Language and Literature, BS Statistics, BS Mathematics, BS Environmental Science, BS Chemistry, BS Biology) of the College of Arts and Sciences were enlisted as participants. Participants will be randomly assigned to the three treatment conditions: (a.1) 1 y/o picture of a female to be paired to four pictures of $21 \mathrm{y} / \mathrm{o}$ female pictures;

$>7 \mathrm{y} / \mathrm{o}$ picture of a female to be paired to four pictures of $21 \mathrm{y} / \mathrm{o}$ female pictures; and

$>12 \mathrm{y} / \mathrm{o}$ picture of a female to be paired to four pictures of $21 \mathrm{y} / \mathrm{o}$.

\subsection{Materials}

Pictures - In this experiment, a total of 21 pictures were used. Seven pictures of a 1 year old, seven picture of 12 year old and also seven pictures of 21 years old, all were pictures of female individuals. The pictures used on female participants were the same of those used on male participants. The researchers prepared 7 pictures of 1 year old, 7 pictures of 12 years old and also 7 pictures of 21 years old. The pictures used in the experiment were adjusted to sepia scale and have uniform sizes. All of the pictures are distinctive Filipino who happens to be twenty-one years old and above so that they have a picture of a twenty-one year old picture. The selected pictures were not similarly dressed and the background is not similar because of limited resources.

Rating Sheet/questionnaire - Aside from the pictures prepared by the researchers, a separate answer sheet was devised and produced which contains numbers that corresponds to the order of pictures in the experiment. The scores of participants were recorded by the researcher on this sheet. In addition, an open-ended question was posed for each item to determine the reason why the specific respondent chose that option.

\subsection{Procedures}

Phase I - Seven (7) pictures of 1 year old, seven pictures of 12 years old and another seven pictures of 21 years old all were female individuals prepared by the researchers and assured that the pictures were similar in color and sizes. Students from the College of Arts and Sciences were enlisted as participants. A total of 54 participants came from nine courses of College of Arts and Sciences, Central Luzon State University, Science City of Munoz, Nueva Ecija, Philippines. Participation is agreed upon by the researchers and the participants.

Phase II - The data gathering was done in a room that is conducive enough for a good ambience of the setting. It was conducted during the second semester of School Year 2014-2015. The experiment was performed 
Dullas, A. R., \& Tolentino, M. N.

for three days of the same hour (morning session) for the reason of having a scheduled day for every treatment level. Each session is composed of 18 participants per day. One by one, each participant is given a time which is five seconds to look at the 1 year old picture, seven (7) years old, and twelve (12) years old pictures before the future picture appears which are paired to twenty-one (21) years old picture. Another 20 seconds per item is given to the participants to choose and finalize his/her answer for the identified future picture of the female photographs. In addition, an open-ended question was posed for each item to determine the reason why the specific respondent chose that option. Upon finalizing the answer, the researchers listed the participants' choice on the score sheet and keep a tally right after all the participants were done answering the experiment.

\subsection{Data Analysis}

The data gathered from the experiment were analyzed using descriptive statistics. The data are also arranged according to the treatment levels (Analysis of Variance $3 \times 2$ between subject design). The researcher utilized SPSS IBM 20 as a tool for the analysis.

\section{Results}

This portion of research tackles the findings and analyses of the study. Analyses focus first on the descriptive statistics of the findings specifically mean and standard deviation. Then it is followed by the inferential statistics on the effect of age interval (picture gap) of the stimuli on picture recognition/identification. Moreover, the differences between the performances of the identifiers' sexual orientation (male, female) on picture recognition are also explored. The interaction between the treatment level and sexual orientation is further investigated.

\section{Table 1}

Participants' distribution on the treatment conditions

\begin{tabular}{lcccc}
\hline & 1 y/o paired to 21 y/o (a1) & 7 y/o paired to 21 y/o (a2) & 12 y/o paired to 21 y/o (a3) \\
\hline Male (b1) & $\mathrm{n}=9$ & $\mathrm{n}=9$ & $\mathrm{n}=9$ \\
Female (b2) & $\mathrm{n}=9$ & $\mathrm{n}=9$ & $\mathrm{n}=9$ \\
\hline Note. $\mathrm{N}=54$ & & &
\end{tabular}

Note. $\mathrm{N}=54$

Table 2

Over-all mean scores and standard deviation of male and female participants

\begin{tabular}{ccccc}
\hline Treatment & Sex & Mean & SD & $\mathrm{n}$ \\
\hline \multirow{3}{*}{ a1-1 yr/o } & Male & 1.89 & 1.05 & 9 \\
& Female & 1.78 & .83 & 9 \\
& Total & 1.83 & .92 & 18 \\
\hline \multirow{3}{*}{ a2-7 yrs/o } & Male & 3.78 & 1.30 & 9 \\
& Female & 3.00 & 1.50 & 9 \\
& Total & 3.39 & 1.42 & 18 \\
\hline \multirow{3}{*}{ 33-12 yrs/o } & Male & 5.44 & .88 & 9 \\
& Female & 6.11 & 1.27 & 9 \\
& Total & 5.78 & 1.11 & 18 \\
\hline \multirow{2}{*}{ Total } & Male & 3.70 & 1.81 & 27 \\
& Female & 3.63 & 2.20 & 27 \\
& Total & 3.67 & 2.00 & 54 \\
\hline
\end{tabular}

On Table 2, findings of the study revealed that in terms of the descriptive statistics, treatment a3 (12 yrs/old) has the highest scores on picture identification with $\mathrm{M}=5.778$; $\mathrm{sd}=1.114$ followed by treatment a2 ( $7 \mathrm{yrs} / \mathrm{o})$ with $\mathrm{M}=3.389$; $\mathrm{sd}=1.419$ and treatment a1 ( $1 \mathrm{yr} / \mathrm{o})$ with score $\mathrm{M}=1.833$; $\mathrm{sd}=.923$ respectively. These findings based on mean scores showed that participants easily recognized pictures as the age of the stimulus are near (or age interval decreases) the target stimulus (21 yrs/o). These results are further substantiated on the analysis of variance ( $3 \times 2$ ANOVA) and post hoc tests. The analysis revealed that there are significant differences between 
The effects of age interval of the stimuli and sexual orientation of the participants on face recognition

three treatment conditions. The main effect showed the difference at 0.05 level of significance with value $F$ (1, 54) $=52.382, p=.000$. See table 3 .

Table 3

Main effects and interaction effect of age and sexual orientation on picture recognition

\begin{tabular}{lcccccc}
\hline \multicolumn{1}{c}{ Source } & $\begin{array}{c}\text { Type III Sum of } \\
\text { Squares }\end{array}$ & df & Mean Square & F & Sig. & $\begin{array}{c}\text { Partial Eta } \\
\text { Squared }\end{array}$ \\
\hline Corrected Model & $146.889^{\mathrm{a}}$ & 5 & 29.378 & 21.657 & .000 & .693 \\
Intercept & 726.000 & 1 & 726.000 & 535.208 & .000 & .918 \\
Treatment & 142.111 & 2 & 71.056 & 52.382 & .000 & .686 \\
Sex & .074 & 1 & .074 & .055 & .816 & .001 \\
treatment $*$ sex & 4.704 & 2 & 2.352 & 1.734 & .187 & .067 \\
Error & 65.111 & 48 & 1.356 & & & \\
Total & 938.000 & 54 & & & & \\
Corrected Total & 212.000 & 53 & & & & \\
a. R Squared $=.693$ (Adjusted R Squared $=.661)$ & &
\end{tabular}

To supplement the differences between the treatment conditions, Tukey HD and Scheffe test are utilized. Analysis showed that treatment condition a3 (12 yrs/old) is significantly different to treatment a2 (7 yrs/o) with mean difference of 2.3889, $s d=.3882, p=.000$ and also significantly different to treatment a1 (1 yr/o) with mean difference of 3.9444, $s d=.3882, p=.000$. Moreover, participants in treatment a2 (7 yrs/o) scored higher in picture recognition than participants in treatment a1 $(1 \mathrm{yr} / \mathrm{o})$ with mean difference of $1.5556, s d=.3882, p=.001$ See Table 4.

Table 4

Multiple comparisons tests on the three treatment conditions

\begin{tabular}{|c|c|c|c|c|c|}
\hline & (I) treatment & $(\mathrm{J})$ treatment & Mean Difference (I-J) & Std. Error & Sig. \\
\hline \multirow{6}{*}{ Tukey HSD } & \multirow[b]{2}{*}{ a1-1 yr/o } & a2-7 yrs/o & $-1.5556^{*}$ & .38823 & .001 \\
\hline & & a3-12 yrs/o & $-3.9444^{*}$ & .38823 & .000 \\
\hline & \multirow{2}{*}{ a2-7 yrs/o } & a1-1 yr/o & $1.5556^{*}$ & .38823 & .001 \\
\hline & & a3-12 yrs/o & $-2.3889^{*}$ & .38823 & .000 \\
\hline & \multirow{2}{*}{ a3-12 yrs/o } & a1-1 yr/o & $3.9444^{*}$ & .38823 & .000 \\
\hline & & a2-7 yrs/o & $2.3889^{*}$ & .38823 & .000 \\
\hline \multirow{6}{*}{ Scheffe } & \multirow{2}{*}{ a1-1 yr/o } & a2-7 yrs/o & $-1.5556^{*}$ & .38823 & .001 \\
\hline & & a3-12 yrs/o & $-3.9444^{*}$ & .38823 & .000 \\
\hline & \multirow{2}{*}{ a2-7 yrs/o } & a1-1 yr/o & $1.5556^{*}$ & .38823 & .001 \\
\hline & & a3-12 yrs/o & $-2.3889^{*}$ & .38823 & .000 \\
\hline & \multirow{2}{*}{ a3-12 yrs/o } & a1-1 yr/o & $3.9444^{*}$ & .38823 & .000 \\
\hline & & a2-7 yrs/o & $2.3889^{*}$ & .38823 & .000 \\
\hline \multirow{6}{*}{ LSD } & \multirow{2}{*}{ a1-1 yr/o } & a2-7 yrs/o & $-1.5556^{*}$ & .38823 & .000 \\
\hline & & a3-12 yrs/o & $-3.9444^{*}$ & .38823 & .000 \\
\hline & \multirow{2}{*}{ a2-7 yrs/o } & a1-1 yr/o & $1.5556^{*}$ & .38823 & .000 \\
\hline & & a3-12 yrs/o & $-2.3889^{*}$ & .38823 & .000 \\
\hline & \multirow{2}{*}{ a3-12 yrs/o } & a1-1 yr/o & $3.9444^{*}$ & .38823 & .000 \\
\hline & & a2-7 yrs/o & $2.3889^{*}$ & .38823 & .000 \\
\hline
\end{tabular}

Note. Based on observed means. The error term is Mean Square(Error) $=1.356 . *$ The mean difference is significant at the .05 level.

The result of the ANOVA accepts the hypothesis that the means of the three treatments varied. Furthermore, the analyses revealed both on descriptive and inferential statistics that as the age of picture gap or the age interval of the stimulus decreases to the target stimulus; the higher the participants scored on picture recognition/identification.

In terms of the mean scores on the performance of male and female participants in picture recognition, results showed that in treatment condition a1, male participants obtained mean score of 1.889 , sd $=1.054$ while female participants got a mean score of $1.778, \mathrm{sd}=0.833$. In treatment condition a2, males scored a mean of 
3.7778, sd $=1.302$ while females had a mean performance of $3.00, \mathrm{sd}=1.500$. And in treatment condition a3, male and female participants gained mean scores of 5.444, $\mathrm{sd}=.882$ and $6.111, \mathrm{sd}=1.269$ respectively. In terms of the overall performance of the participants in picture recognition, males $(\mathrm{M}=3.704$, sd $=1.815)$ slightly outperformed females $(\mathrm{M}=3.629$, sd = 2.204). While the mean scores of the participants in terms of the sexual orientation slightly varied, result of the ANOVA showed that there is no significant difference $(F(1,54)=.055$, $p=.816$ ) between males and females on picture recognition/identification performance.

In addition, the data on the table 3 showed the treatment combination or the interaction between the independent variables, the picture gap and the sex of the participants. The analysis showed that there is no interaction effect between the treatment level (a1,a2,a3) and the sexual orientation of the participants (b1 and b2) on picture recognition/identification $(F(1,54)=1.754, p=.187$. On the other hand, answers to open-ended question ("What is your reason for choosing that specific option") reveal similar responses such as: "facial features matches like the nose, eyes, shape of the face"; "the way the curvature of the lips or their smile are similar"; "their features are similar".

\section{Discussion}

This part includes the implication and integration of the data and findings of other related studies. The experimental study have validated of what literature claims that as the age difference between pairs of images (with age intervals) increases, the proportion of images with high similarity scores (if there are participants assigned to match the facial images) decreases. Furthermore, the result of the ANOVA accepts the hypothesis that the means of the three treatments varied. This can be observed with the raw mean scores with each treatment level. The treatment condition a1, having the age interval of 20 years, was figured out to be the most difficult pairing with the mean (participants' correct recognition). This confirmed the study made by Chellappa (2007) that from zero to eighteen years face recognition across age progression has an added difficulty as the facial features naturally experience drastic changes. In addition, there are also significant differences between treatment conditions a3 and a 2 having an interval of 14 years. This result is supported by Multiple Comparisons Tests- Tukey HD and Scheffe Tests.

This is further verified by result of Analysis of variance that there are significant differences on the treatment conditions $(F(1,54)=52.382, p=.000)$. It is, however, important to mention and discuss such observations as they are supported by results of previous studies.

On the other hand, it is not important to consider whether one is male or female in terms of recognizing or identifying picture because base from the result of the experiment, sexual orientation does not have effect in terms of identifying picture. This finding is actually one of the major contributions of the study in which this area is still lacking of. Moreover, interaction between sexual orientation and the treatment is also irrelevant as the result of this study. This is also another highlight that when sexual orientation of the identifier is paired to treatment, the effect is nearly negligible. Moreover, these findings reject both hypotheses of the research that (1) there is significant difference on sexual orientation of the participants in terms of recognizing picture and (2) that there are interaction effects between the treatment levels - that is the picture gap or interval in years in identifying pictures and the sexual orientation of the participants.

\section{Conclusion and Recommendation}

The respondents face recognition was based on the components or facial features of the stimulus not on the global features of the stimulus. This was substantiated to their responses on the open-ended question: What is your reason for choosing that specific option? This reveals a similar answer, which is based on components or facial features of the target stimuli. Using the Biederman's Recognition By Components Theory (1987), this may imply that recognition by object including facial recognition across age progression was guided by the recognition not on the complete object but the object's components which are combinations of the geons 
The effects of age interval of the stimuli and sexual orientation of the participants on face recognition

(Biederman, 1987) which in this case the combination of the different facial features of the target stimuli. The findings was also further substantiated that in terms of component versus general approaches on face recognition, component system of face recognition outperformed general based system of face recognition (Heisele et. al., 2003). These findings imply that the identifiers successfully recognize the target stimuli by looking at the specific features (such as facial features like the nose, eyes, shape of the face"; "the way the curvature of the lips or their smile are similar"; "their features are similar).

Through the research results and procedure, the researcher has formulated the following recommendations. (1) Further researches may use interval periods of equal year gaps, but with each interval period being paired with one specific age. (i.e., 6year-old, 8, 10,12,14,16, 18, 20, image paired with a 22-year old image). (2) Further experiments may make use of both male and female images as their instruments so as to see the difference, if there be any, on recognizing faces of both sexes. (3) Further exploration on the effects of facial features on face recognition across age progression. This was on the reason that in the current study, only open-ended question was used to verify their reason on selecting the target stimulus.

In terms of the scope and limitations of the study, this study is only focused on the effect of picture gap (age interval) in terms of recognizing picture. Moreover, it only looked on the difference between male and female in recognizing/identifying pictures. Furthermore, exploration on the effects of facial features on face recognition across age progression was surveyed using open-ended question- e.i. verifying their reason on why they select that target stimulus. Another major limitation of study is the sample. The study only utilized adolescents ages 16 to 21 . Thus, it is also further recommended to used children, middle-age and old age respondents using the variable used in the current study.

\section{References}

Anastasi, J., \& Rhodes, M. (2005). An own-age bias in face recognition for children and older adults. Psychonomic Bulletin \& Review, 12(6), 1043-1047. http://dx.doi.org/10.3758/BF03206441

Arat, M., Koklu, A., Ozdiler, E., Rubenduz, M., \& Erdogan, B. (2001). Craniofacial growth and skeletal maturation: A mixed longitudinal study. European Journal of Orthodontics, 23(4), 355-361. http://dx.doi.org/10.1093/ejo/23.4.355

Biederman, I. (1987). Recognition-by-components: A theory of human image understanding. Psychological Review, 94(2), 115-147. http://dx.doi.org/10.1037/0033-295X.94.2.115

Chellappa, R. (2007). Trends in face recognition [PowerPoint slides]. Retrieved from http://www.noblis.org/Business_Areas/NationalHomelandSecurity/BIC_Trends_in_Face_Recognition.pd $\underline{\mathrm{f}}$

Heisele, B., Ho, P., Wu, J., \& Poggio, T. (2003). Face recognition: Component-based versus global approached. Computer Vision and Image Understanding, 91(1-2), 6-21. http://dx.doi.org/10.1016/S1077-3142(03)00073-0

Lamont, A. C., Stewart-Williams, S., \& Podd, J. (2005). Face recognition and aging: Effects of target age and memory load. Memory and Cognition, 33(6), 1017-1024. http://dx.doi.org/10.3758/BF03193209

Ling, H., Soatto, S., Ramanathan, N., \& Jacobs, D. (2007). A study of face recognition as people age. Retrieved from http://www.cfar.umd.edu/ ramanath/Ramanathan_iccv2007.pdf http://dx.doi.org/10.1109/iccv.2007.4409069

Mason, S. E. (1986). Age and gender as factors in facial recognition and identification. Experimental Aging Research, 12(3), 151-154. http://dx.doi.org/10.1080/03610738608259453

Ramanathan, N., \& Chellappa, R. (2005). Face verification across age progression. In 2005 IEEE Computer society conference on computer vision and pattern recognition (vol. 2, pp. 462-469). IEEE. http://dx.doi.org/10.1109/CVPR.2005.153

Ramanathan, N., \& Chellappa, R. (2006). Face verification across age progression. Retrieved from http://www.cfar.umd.edu/ ramanath/Ramanathan_ip2006.pdf 
Dullas, A. R., \& Tolentino, M. N. 